\title{
A simple model of trees for unicellular maps
}

\author{
Guillaume Chapuy 1 |and Valentin Féray非and Éric Fusy ${ }^{3 \dagger}$ \\ ${ }^{1}$ CNRS, LIAFA - UMR 7089, Université Paris 7, Paris, France \\ ${ }^{2}$ CNRS, LaBRI - UMR 5800, Université de Bordeaux, Talence, France \\ ${ }^{3}$ CNRS, LIX - UMR 7161, École Polytechnique, Palaiseau, France
}

\begin{abstract}
We consider unicellular maps, or polygon gluings, of fixed genus. In FPSAC '09 the first author gave a recursive bijection transforming unicellular maps into trees, explaining the presence of Catalan numbers in counting formulas for these objects. In this paper, we give another bijection that explicitly describes the "recursive part" of the first bijection. As a result we obtain a very simple description of unicellular maps as pairs made by a plane tree and a permutation-like structure. All the previously known formulas follow as an immediate corollary or easy exercise, thus giving a bijective proof for each of them, in a unified way. For some of these formulas, this is the first bijective proof, e.g. the Harer-Zagier recurrence formula, or the Lehman-Walsh/Goupil-Schaeffer formulas. Thanks to previous work of the second author this also leads us to a new expression for Stanley character polynomials, which evaluate irreducible characters of the symmetric group.
\end{abstract}

Résumé. Nous considérons des cartes orientées à une face de genre fixé. À SFCA'09 le premier auteur a introduit une bijection récursive envoyant une carte unicellulaire vers un arbre, ce qui permet d'obtenir des formules énumératives pour les cartes à une face (et en particulier la présence des nombres de Catalan). Dans l'article ici présent, et en nous appuyant sur la bijection ci-dessus, nous obtenons une incarnation très simple des cartes à une face comme des paires formées d'un arbre plan et d'une permutation d'un certain type. Toutes les formules précédemment connues découlent aisément de cette nouvelle incarnation, donnant des preuves bijectives dans un cadre unifié. Pour certaines de ces formules, telles que la récurrence de Harer-Zagier ou les formules de Lehman-Walsh/Goupil-Schaeffer, nous obtenons la première preuve bijective connue. Par ailleurs, en combinant notre approche avec des travaux du second auteur, nous obtenons une nouvelle expression pour les polynômes de Stanley qui donnent certaines évaluations des caractères du groupe symétrique.

Keywords: one-face map, Stanley character polynomial, bijection, Harer-Zagier formula, Rémy's bijection.

\section{Introduction}

A unicellular map is a connected graph embedded in a surface in such a way that the complement of the graph is a topological disk. These objects have appeared frequently in combinatorics in the last forty years, in relation with the general theory of map enumeration, but also with the representation theory of the symmetric group, the study of permutation factorizations, or the study of moduli spaces of curves. All

\footnotetext{
${ }^{\dagger}$ Partially supported by the ERC grant StG 208471 - ExploreMaps.

$\ddagger$ Partially supported by ANR project PSYCO 11-JS02-001 
these connections have turned the enumeration of unicellular maps into an important research field (for the many connections with other areas, see [9] and references therein; for an overview of the results see the introductions of the papers [3, 1]). The main results in the domain can be roughly separated in two families.

The first family deals with colored maps (maps endowed with an application from its vertex set to a set of $q$ colors). This implies "summation" enumeration formulas (see [8, 14, 11] or paragraph 3.4 below). These formulas are often elegant, and different combinatorial proofs for them have been given in the past few years [10, 6, 14, 11, 1]. The issue is that some important topological information, such as the genus of the surface, is not apparent in these constructions.

Formulas of the second family keep track explicitly of the genus of the surface. This includes inductive relations (like the Harer-Zagier recurrence formula [8]) or explict (but quite involved) closed forms (Lehman-Walsh [15] and Goupil-Schaeffer [7] formulas). From a combinatorial point of view, these formulas are harder to understand. A step in this direction was done by the first author in [3] (this construction is explained in subsection 2.2, which led to new inductions relations and to new formulas. However the link with other formulas in the second family remained mysterious, and [3] left open the problem of finding combinatorial proofs of these formulas.

The goal of this paper is to present a new bijection between unicellular maps and surprisingly simple objects which we call $C$-decorated trees (these are merely plane trees equipped with a certain kind of permutation on their vertices). This bijection is based on the previous work of the first author [3]: we explicitly describe the "recursive part" appearing in this work. As a consequence, not only can we reprove all the aforementioned formulas in a bijective way, thus giving the first bijective proof for several of them, but we do that in unified way. Indeed, C-decorated trees are so simple combinatorial objects that all formulas follow from our bijection as an immediate corollary or easy exercise.

Another interesting application of this bijection is a new explicit way of computing the so-called Stanley character polynomials, which are nothing but the evaluation of irreducible characters of the symmetric groups, properly normalized and parametrized. Indeed, in a previous work [4], the second author expressed these polynomials as a generating function of (properly weighted) unicellular maps. Although we do not obtain a "closed form" expression (there is no reason to believe that such a form exists!), we express Stanley character polynomials as the result of a term-substitution in free cumulants, which are another meaningful quantity in representation theory of symmetric groups.

\section{The main bijection}

\subsection{Unicellular maps and C-decorated trees}

A map $M$ of genus $g \geq 0$ is a connected graph $G$ embedded on a closed compact oriented surface $S$ of genus $g$, such that $S \backslash G$ is a collection of topological disks, which are called the faces of $M$. Loops and multiple edges are allowed. The graph $G$ is called the underlying graph of $M$ and $S$ its underlying surface. Two maps that differ only by an oriented homeomorphism between the underlying surfaces are considered the same. A corner of $M$ is the angular sector between two consecutive edges around a vertex. A rooted map is a map with a marked corner, called the root; the vertex incident to the root is called the root-vertex. From now on, all maps are assumed to be rooted (note that the underlying graph of a rooted map is naturally vertex-rooted). A unicellular map is a map with a unique face. The classical Euler relation $|V|-|E|+|F|=2-2 g$ ensures that a unicellular map with $n$ edges has $n+1-2 g$ vertices. A plane tree is a unicellular map of genus 0 . 

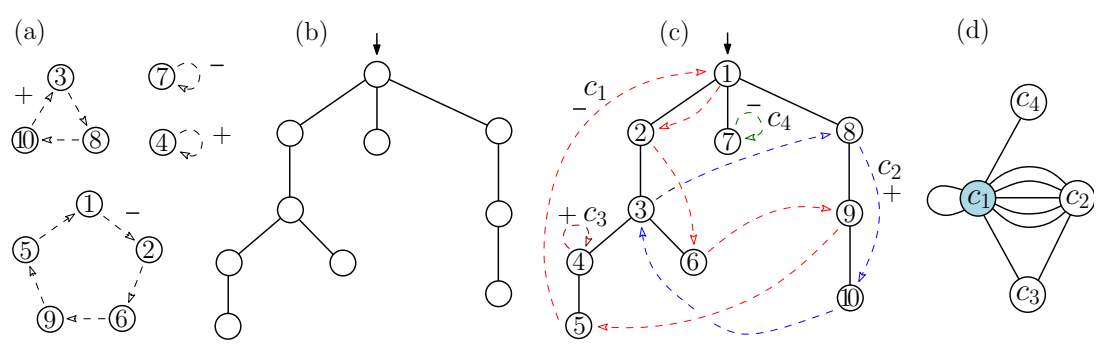

Fig. 1:

(a) A C-permutation $\sigma$.

(b) A plane tree $T$.

(c) The C-decorated tree $(T, \sigma)$.

(d) The underlying graph of $(T, \sigma)$.

A rotation system on a connected graph $G$ consists in a cyclic ordering of the half-edges of $G$ around each vertex. Given a map $M$, its underlying graph $G$ is naturally equipped with a rotation system given by the clockwise ordering of half-edges on the surface in a vicinity of each vertex. It is well-known that this correspondence is 1-to-1, i.e. a map can be considered as a connected graph equipped with a rotation system (thus, as a purely combinatorial object). We will take this viewpoint from now on.

A cycle-signed permutation is a permutation where each cycle carries a sign, either + or - . A $C$ permutation is a cycle-signed permutation where all cycles have odd length, see Figure 11 a). For each C-permutation $\sigma$ on $n$ elements, the rank of $\sigma$ is defined as $r(\sigma)=n-\ell(\sigma)$, where $\ell(\sigma)$ is the number of cycles of $\sigma$. Note that $r(\sigma)$ is even since all cycles have odd length. The genus of $\sigma$ is defined as $r(\sigma) / 2$. A $C$-decorated tree on $n$ edges is a pair $\gamma=(T, \sigma)$ where $T$ is a plane tree with $n$ edges and $\sigma$ is a C-permutation of $n+1$ elements. The genus of $\gamma$ is defined to be the genus of $\sigma$. Note that the $n+1$ vertices of $T$ can be canonically numbered from 1 to $n+1$ (e.g., following a left-to-right depth-first traversal), hence $\sigma$ can be seen as a permutation of the vertices of $T$, see Figure 1(c). The underlying graph of $\gamma$ is the (vertex-rooted) graph $G$ obtained from $T$ by merging into a single vertex the vertices in each cycle of $\sigma$ (so that the vertices of $G$ correspond to the cycles of $\sigma$ ), see Figure11(d).

Definition 1 For $n, g$ nonnegative integers, denote by $\mathcal{E}_{g}(n)$ the set of unicellular maps of genus $g$ with $n$ edges; and denote by $\mathcal{T}_{g}(n)$ the set of $C$-decorated trees of genus $g$ with $n$ edges.

For $\mathcal{A}$ a finite set, $k \mathcal{A}$ denotes the set made of $k$ disjoint copies of $\mathcal{A}$. For two finite sets $\mathcal{A}$ and $\mathcal{B}$, we write $\mathcal{A} \simeq \mathcal{B}$ if there is a bijection between $\mathcal{A}$ and $\mathcal{B}$. Our main result will be to show that $2^{n+1} \mathcal{E}_{g}(n) \simeq \mathcal{T}_{g}(n)$, with a bijection which preserves the underlying graphs of the objects.

\subsection{Recursive decomposition of unicellular maps}

In this section, we briefly recall a combinatorial method developed in [3] to decompose unicellular maps.

Proposition 1 (Chapuy [3]) For $k \geq 1$, denote by $\mathcal{E}_{g}^{(2 k+1)}(n)$ the set of maps from $\mathcal{E}_{g}(n)$ in which a set of $2 k+1$ vertices is distinguished. Then for $g>0$ and $n \geq 0$,

$$
2 g \mathcal{E}_{g}(n) \simeq \mathcal{E}_{g-1}^{(3)}(n)+\mathcal{E}_{g-2}^{(5)}(n)+\mathcal{E}_{g-3}^{(7)}(n)+\cdots+\mathcal{E}_{0}^{(2 g+1)}(n) .
$$

In addition, if $M$ and $\left(M^{\prime}, S^{\prime}\right)$ are in correspondence, then the underlying graph of $M$ is obtained from the underlying graph of $M^{\prime}$ by merging the vertices in $S^{\prime}$ into a single vertex.

We now sketch briefly the construction of [3]. Although this is not really needed for the sequel, we believe that it gives a good insight into the objects we are dealing with (readers in a hurry may take Proposition 1 for granted and jump directly to subsection 2.3. We refer to [3] for proofs and details. 


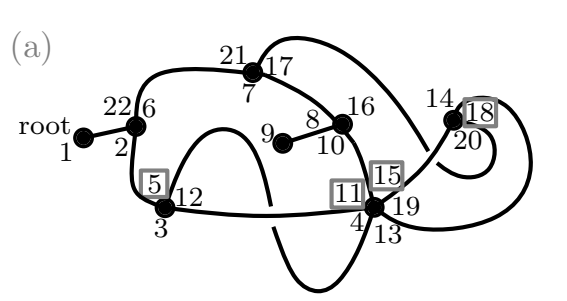

(c) if $c_{3}$ has minimum label in $v_{3}$ : stop. else: $-c_{3}$ is a trisection of the new map.

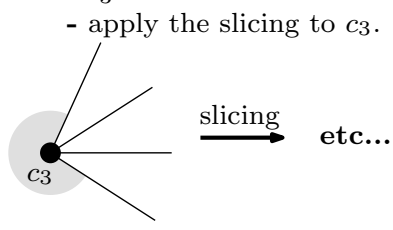

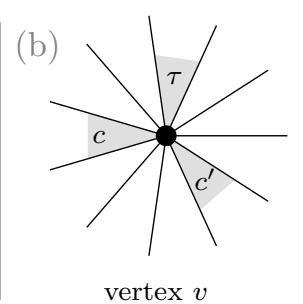
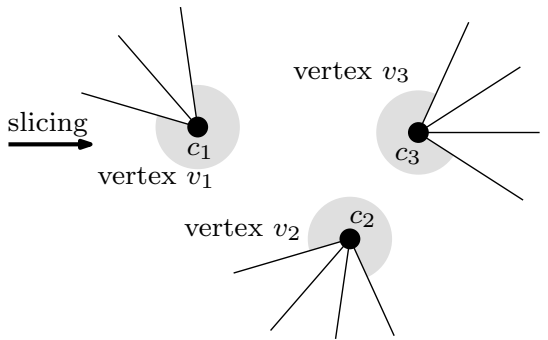

Fig. 2: (a) A unicellular map of genus 2 equipped with its corner labelling. Labels corresponding to trisections are boxed. (b) Given a trisection $\tau$, two other corners of interest $c$ and $c^{\prime}$ are canonically defined (see text). "Slicing the trisection" then gives rise to three new vertices $v_{1}, v_{2}, v_{3}$, with distinguished corners $c_{1}, c_{2}, c_{3}$. (c) The recursive procedure of [3]: if $c_{3}$ is the minimum corner of $v_{3}$, then stop; else, as shown in [3], $c_{3}$ is a trisection of the new map $M^{\prime}$ : in this case, iterate the slicing operation on $\left(M^{\prime}, c_{3}\right)$.

We first explain where the factor $2 g$ comes from in (1). Let $M$ be a rooted unicellular map of genus $g$ with $n$ edges. Then $M$ has $2 n$ corners, and we label them from 1 to $2 n$ incrementally, starting from the root, and going clockwise around the (unique) face of $M$ (Figure 2). Let $v$ be a vertex of $M$, let $k$ be its degree, and let $\left(a_{1}, a_{2}, \ldots, a_{k}\right)$ be the sequence of the labels of corners incident to it, read in counterclockwise direction around $v$ starting from the minimal label $a_{1}=\min a_{i}$. If for some $j \in$ $\llbracket 1, k-1 \rrbracket$, we have $a_{j+1}<a_{j}$, we say that the corner of $v$ labelled by $a_{j+1}$ is a trisection of $M$. Figure 2(a) shows a map of genus two having four trisections. More generally we have:

Lemma 2 ([3]) A unicellular map of genus $g$ contains exactly $2 g$ trisections. In other words, the set of unicellular maps of genus $g$ with $n$ edges and a marked trisection is isomorphic to $2 g \mathcal{E}_{g}(n)$.

Now, let $\tau$ be a trisection of $M$ of label $l(\tau)$, and let $v$ the vertex its belongs to. We denote $c$ the corner of $v$ with minimum label and $c^{\prime}$ the corner with minimum label among those which appear between $c$ and $\tau$ clockwise around $v$ and whose label is greater than $l(\tau)$. By definition of a trisection, $c^{\prime}$ is well defined. We then construct a new map $M^{\prime}$, by slicing the vertex $v$ into three new vertices using the three corners $c, c^{\prime}, \tau$ as on Figure 2 (b). We say that the map $M^{\prime}$ is obtained from $M$ by slicing the trisection $\tau$. As shown in [3], the new map $M^{\prime}$ is a unicellular map of genus $g-1$. We can thus relabel the $2 n$ corners of $M^{\prime}$ from 1 to $2 n$, according to the procedure we already used for $M$. Among these corners, three of them, say $c_{1}, c_{2}, c_{3}$ are naturally inherited from the slicing of $v$, as on Figure 2(b). Let $v_{1}, v_{2}, v_{3}$ be the vertices they belong to, respectively. Then the following is true [3]: In the map $M^{\prime}$, the corner $c_{i}$ has the smallest label around the vertex $v_{i}$, for $i \in\{1,2\}$. For $i=3$, either the same is true, or $c_{3}$ is a trisection of the map $M^{\prime}$.

We now finally describe the bijection promised in Proposition 1 . It is defined recursively on the genus, as follows. Given a map $M \in \mathcal{E}_{g}(n)$ with a marked trisection $\tau$, let $M^{\prime}$ be obtained from $M$ by the slicing of $\tau$, and let $c_{i}, v_{i}$ be defined as above for $i \in\{1,2,3\}$. If $c_{3}$ has the minimum label in $v_{3}$, set $\Psi(M, \tau):=\left(M^{\prime},\left\{v_{1}, v_{2}, v_{3}\right\}\right)$, which is an element of $\mathcal{E}_{g-1}^{(3)}(n)$. Else, let $\left(M^{\prime \prime}, S\right)=\Psi\left(M^{\prime}, c_{3}\right)$, and set $\Psi(M, \tau):=\left(M^{\prime \prime}, S \cup\left\{v_{1}, v_{2}\right\}\right)$. Note that this recursive algorithm necessarily stops, since the genus of the map decreases and since there are no trisections in unicellular maps of genus 0 (plane trees). Thus this procedure yields recursively a mapping that associates to a map $M$ with a marked trisection $\tau$ another 
map $M^{\prime \prime}$ of a smaller genus, with a set $S^{\prime \prime}$ of marked vertices (namely the set of vertices which have been involved in a slicing at some point of the procedure). The set $S^{\prime \prime}$ of marked vertices necessarily has odd cardinality, as easily seen by induction. Moreover, it is clear that the underlying graph of $M$ coincides with the underlying graph of $M^{\prime \prime}$ in which the vertices of $S^{\prime \prime}$ have been identified together into a single vertex. One can show that $\Psi$ is a bijection by constructing explicitly the inverse mapping [3].

\subsection{Recursive decomposition of C-decorated trees}

We now propose a recursive method to decompose C-decorated trees, which can be seen as parallel to the decomposition of unicellular maps given in the previous section. Denote by $\mathcal{C}(n)$ (resp. $\mathcal{C}_{g}(n)$ ) the set of C-permutations on $n$ elements (resp. on $n$ elements and of genus $g$ ). A signed sequence of integers is a pair $(\epsilon, S)$ where $S$ is an integer sequence and $\epsilon$ is a sign, either + or -.

Lemma 3 Let $X$ be a finite non-empty set of positive integers. Then there is a bijection between signed sequences of distinct integers from $X$-all elements of $X$ being present in the sequence-and $C$ permutations on the set $X$. In addition the $C$-permutation has one cycle if and only if the signed sequence has odd length and starts with its minimal element.

Proof: Let $\gamma$ be a signed sequence, e.g. $\gamma={ }^{+}(4731562)$. If $\gamma$ has odd length and starts with its minimal element, return $\gamma$ seen as a unicyclic C-permutation (where the unique cycle is written sequentially). Otherwise cut $\gamma$ as $\gamma=\gamma_{1} \gamma_{2}$, where $\gamma_{2}$ starts with the minimal element in $\gamma$ (in our example, $\gamma_{1}={ }^{+}(473)$ and $\gamma_{2}=(1562)$ ). If $\gamma_{2}$ has odd length, then "produce" the signed cycle ${ }^{+} \gamma_{2}$. If $\gamma_{2}$ has even length, move the second element of $\gamma_{2}$ to the end of $\gamma_{1}$, and "produce" the signed cycle ${ }^{-} \gamma_{2}$. Then (in both cases), restart the same process on $\gamma=\gamma_{1}$, producing one (signed) cycle at each step, until $\gamma$ is odd and starts with its minimal element, in which case one produces $\gamma$ as the last signed cycle. (In our example, the signed cycles successively produced are ${ }^{-}(162),-(3)$, and ${ }^{+}(475)$.) The process clearly yields a collection of signed cycles of odd lengths, i.e., yields a C-permutation. The mapping is easy to invert (we omit details in this extended abstract), so it gives a bijection.

An element of a C-permutation is called non-minimal if it is not the minimum in its cycle. Non-minimal elements play the same role for $\mathrm{C}$-permutations (and C-decorated trees) as trisections for unicellular maps. Indeed, a C-permutation of genus $g$ has $2 g$ non-minimal elements (compare with Lemma 2), and moreover we have the following analogue of Proposition 1 .

Proposition 4 For $k \geq 1$, denote by $\mathcal{T}_{g}^{(2 k+1)}(n)$ the set of $C$-decorated trees from $\mathcal{T}_{g}(n)$ in which a set of $2 k+1$ cycles is distinguished. Then for $g>0$ and $n \geq 0$,

$$
2 g \mathcal{T}_{g}(n) \simeq \mathcal{T}_{g-1}^{(3)}+\mathcal{T}_{g-2}^{(5)}+\mathcal{T}_{g-3}^{(7)}+\cdots
$$

In addition, if $\gamma$ and $\left(\gamma^{\prime}, S^{\prime}\right)$ are in correspondence, then the underlying graph of $\gamma$ is obtained from the underlying graph of $\gamma^{\prime}$ by merging the vertices corresponding to cycles from $S^{\prime}$ into a single vertex.

Proof: For $k \geq 1$ let $\mathcal{C}_{g}^{(2 k+1)}(n)$ be the set of C-permutations from $\mathcal{C}_{g}(n)$ where a subset of $2 k+1$ cycles are marked. Let $\mathcal{C}_{g}^{\circ}(n)$ be the set of C-permutations from $\mathcal{C}_{g}(n)$ where a non-minimal element is marked. Note that $\mathcal{C}_{g}^{\circ}(n) \simeq 2 g \mathcal{C}_{g}(n)$ since a C-permutation in $\mathcal{C}_{g}(n)$ has $2 g$ non-minimal elements. Moreover $\mathcal{C}_{g}^{\circ}(n) \simeq \sum_{k \geq 1} \mathcal{C}_{g-k}^{(2 k+1)}(n)$ (apply Lemma 3 to the cycle - represented as a signed sequence- 
containing the marked non-minimal element; this produces a collection of $(2 k+1) \geq 3$ signed cycles of odd length, which we take as the marked cycles). Hence $2 g \mathcal{C}_{g}(n) \simeq \sum_{k \geq 1} \mathcal{C}_{g-k}^{(2 k+1)}(n)$. Since $\mathcal{T}_{g}(n)=$ $\mathcal{E}_{0}(n) \times \mathcal{C}_{g}(n+1)$, we conclude that $2 g \mathcal{T}_{g}(n) \simeq \sum_{k \geq 1} \mathcal{T}_{g-k}^{(2 k+1)}(n)$. The statement on the underlying graph just follows from the fact that the procedure in Lemma 3 merges the marked cycles into a unique cycle.

\subsection{The main result}

Theorem 5 For each non-negative integers $n$ and $g$ we have

$$
2^{n+1} \mathcal{E}_{g}(n) \simeq \mathcal{T}_{g}(n)
$$

In addition the cycles of a $C$-decorated tree naturally correspond to the vertices of the associated unicellular map, in such a way that the respective underlying graphs are the same.

Proof: The proof is a simple induction on $g$, whereas $n$ is fixed. The case $g=0$ is obvious. Let $g>0$. The induction hypothesis ensures that for each $g^{\prime}<g, 2^{n+1} \mathcal{E}_{g^{\prime}}^{(2 k+1)}(n) \simeq \mathcal{T}_{g^{\prime}}^{(2 k+1)}(n)$, where the underlying graphs (taking marked vertices vertices into account) of corresponding objects are the same. Hence, by Propositions 1 and 4 , we have $2 g 2^{n+1} \mathcal{E}_{g}(n) \simeq 2 g \mathcal{T}_{g}(n)$, where the underlying graphs of corresponding objects are the same. Finally, one can extract from this $2 g$-to- $2 g$ correspondence a 1 -to- 1 correspondence, which still preserves underlying graphs: think of extracting a perfect matching from a $2 g$-regular bipartite graph, which is possible according to Hall's marriage theorem. Hence $2^{n+1} \mathcal{E}_{g}(n) \simeq \mathcal{T}_{g}(n)$.

\subsection{A fractional, or stochastic, formulation}

Even if this does not hinder enumerative applications to be detailed in the next section, we do not know of an effective (polynomial-time) way to implement the bijection of Theorem 5 , indeed the last step of the proof is to extract a perfect matching from a $2 g$-regular bipartite graph whose size is exponential in $n$.

What can be done effectively is a fractional formulation of the bijection. For a finite set $X$, let $\mathbb{C}\langle X\rangle$ be the set of linear combinations of the form $\sum_{x \in X} u_{x} \cdot x$, where the $x \in X$ are seen as independent formal vectors, and the coefficients $u_{x}$ are in $\mathbb{C}$. Let $\mathbb{R}_{1}^{+}\langle X\rangle \subset \mathbb{C}\langle X\rangle$ be the subset of linear combinations where the coefficients are nonnegative and add up to 1 . Denote by $\mathbf{1}_{X}$ the vector $\sum_{x \in X} x$. For two finite sets $X$ and $Y$, a fractional mapping from $X$ to $Y$ is a linear mapping $\varphi$ from $\mathbb{C}\langle X\rangle$ to $\mathbb{C}\langle Y\rangle$ such that the image of each $x \in X$ is in $\mathbb{R}_{1}^{+}\langle Y\rangle$; the subset of elements of $Y$ with strictly positive coefficients in $\varphi(x)$ is called the image-support of $x$. Note that $\varphi(x)$ identifies to a probability distribution on $Y$; a "call to $\varphi(x)$ " is meant as picking up $y \in Y$ under this distribution. A fractional mapping is bijective if $\mathbf{1}_{X}$ is mapped to $\mathbf{1}_{Y}$, and is deterministic if each $x \in X$ is mapped to some $y \in Y$. Note that, if there is a fractional bijection from $X$ to $Y$, then $|X|=|Y|$ (indeed in that case the matrix of $\varphi$ is bistochastic).

One can now formulate by induction on the genus an effective (the cost of a call is $O(g n)$ ) fractional bijection from $2^{n+1} \mathcal{E}_{g}(n)$ to $\mathcal{T}_{g}(n)$, and similarly from $\mathcal{T}_{g}(n)$ to $2^{n+1} \mathcal{E}_{g}(n)$. The crucial property is that, for $k \geq 1$ and $E, F$ finite sets, if there is a fractional bijection $\Phi$ from $k E$ to $k F$ then one can effectively derive from it a fractional bijection from $E$ to $F$ : map each $x \in E$ to $\frac{1}{k}\left(\iota\left(\Phi\left(x_{1}\right)\right)+\cdots+\iota\left(\Phi\left(x_{k}\right)\right)\right)$, where $x_{1}, \ldots, x_{k}$ are the representatives of $x$ in $k E$, and where $\iota$ is the projection from $k F$ to $F$. Hence by induction on $g$, Propositions 1 and 4 (where the stated combinatorial isomorphisms are effective) 
ensure that there is an effective fractional bijection from $2^{n+1} \mathcal{E}_{g}(n)$ to $\mathcal{T}_{g}(n)$ and similarly from $\mathcal{T}_{g}(n)$ to $2^{n+1} \mathcal{E}_{g}(n)$, such that if $\gamma^{\prime}$ is in the image-support of $\gamma$ then the underlying graphs of $\gamma$ and $\gamma^{\prime}$ are the same.

Note that, given an effective fractional bijection between two sets $X$ and $Y$, and a uniform random sampling algorithm on the set $X$, one obtains immediately a uniform random sampling algorithm for the set $Y$. In the next section, we will use our bijection to prove several enumerative formulas for unicellular maps, starting from elementary results on the enumeration of trees or permutations. In all cases, we will also be granted with a uniform random sampling algorithm for the corresponding unicellular maps, though we will not emphasize this point in the rest of the paper.

\section{Counting formulas for unicellular maps}

It is quite clear that $\mathrm{C}$-decorated trees are much simpler combinatorial objects than unicellular maps. In this section, we use them to give bijective proofs of several known formulas concerning unicellular maps. We focus on the Lehman-Walsh and Goupil-Schaeffer formulas, and the Harer-Zagier recurrence, of which bijective proofs were long-awaited. We also sketch a bijective proof of the Harer-Zagier summation formula (prototype for a family of formulas for which bijective proofs were already known). We insist on the fact that all these proofs are elementary consequences of our main bijection (Theorem 5 .

\subsection{Two immediate corollaries}

The set $\mathcal{T}_{g}(n)=\mathcal{E}_{0}(n) \times \mathcal{C}_{g}(n+1)$ is the product of two sets that are easy to count. Precisely, let $\epsilon_{g}(n)=\left|\mathcal{E}_{g}(n)\right|$ and $c_{g}(n)=\left|\mathcal{C}_{g}(n)\right|$. Recall that $\epsilon_{0}(n)=\operatorname{Cat}(n)$, where $\operatorname{Cat}(n):=\frac{(2 n) !}{n !(n+1) !}$ is the $n$th Catalan number. Therefore Theorem 5 gives $\epsilon_{g}(n)=2^{-n-1} \operatorname{Cat}(n) c_{g}(n+1)$.

It is immediate to give for $c_{g}(n+1)$ a closed form (by summing over all possible cycle types) or an explicit generating series. This yields two classical results for the enumeration of unicellular maps.

For $\gamma=\left(\gamma_{1}, \ldots, \gamma_{\ell}\right)=1^{m_{1}} \ldots k^{m_{k}}$ a partition of $g$, the number $a_{\gamma}(n+1)$ of permutations of $n+1$ elements with cycle-type equal to $1^{n+1-2 g-\ell} 3^{m_{1}} \ldots(2 k+1)^{m_{k}}$ is classically given by the quotient $a_{\gamma}(n+1)=(n+1) ! /\left((n+1-2 g-\ell) ! \prod_{i} m_{i} !(2 i+1)^{m_{i}}\right)$, and the number of C-permutations with this cycle-type is just $a_{\gamma}(n+1) 2^{n+1-2 g}$ (since each cycle has 2 possible signs). Hence, we get the equality $c_{g}(n+1)=2^{n+1-2 g} \sum_{\gamma \vdash g} a_{\gamma}(n+1)$. We thus recover:

Proposition 6 (Walsh and Lehman [15]) The number $\epsilon_{g}(n)$ is given by

$$
\epsilon_{g}(n)=\frac{(2 n) !}{n !(n+1-2 g) ! 2^{2 g}} \sum_{\gamma \vdash g} \frac{(n+1-2 g)_{\ell}}{\prod_{i} m_{i} !(2 i+1)^{m_{i}}}
$$

where $(x)_{k}=\prod_{j=0}^{k-1}(x-j)$, $\ell$ is the number of parts of $\gamma$, and $m_{i}$ is the number of parts of length $i$ in $\gamma$.

The exponential generating function $C(x, y):=\sum_{n, g} \frac{1}{(n+1) !} c_{g}(n+1) y^{n+1} x^{n+1-2 g}$ of signed cycles of odd length ( $y$ marks the number of elements, which are labelled, and $x$ marks the number of cycles) is

$$
C(x, y)=\exp \left(2 x \sum_{k \geq 1} \frac{y^{2 k+1}}{2 k+1}\right)-1=\exp \left(x \log \left(\frac{1+y}{1-y}\right)\right)-1=\left(\frac{1+y}{1-y}\right)^{x}-1 .
$$

Since $c_{0}(1)=2$ and $\frac{1}{(n+1) !} c_{g}(n+1)=\frac{2^{n+1} n !}{(2 n) !} \epsilon_{g}(n)=\frac{2}{(2 n-1) ! !} \epsilon_{g}(n)$ for $n \geq 1$, we recover: 

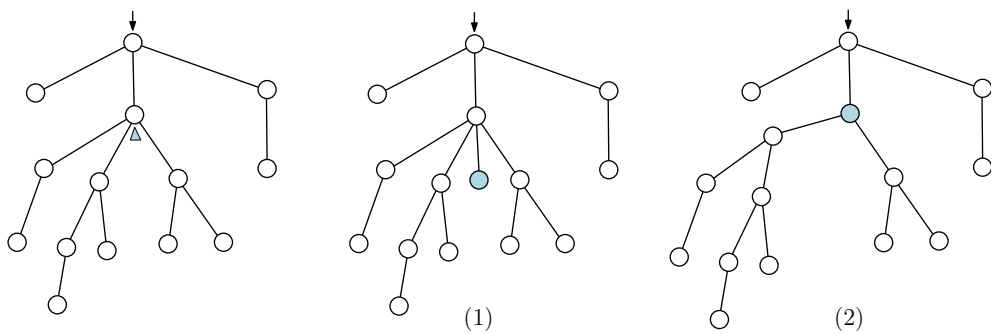

Fig. 3: Rémy's procedure gives two ways to obtain a plane tree with $n$ edges and a marked vertex $v$ from a plane tree with $n-1$ edges and a marked corner: (1) in the first way $v$ is a leaf, (2) in the second way $v$ is a non-leaf.

\section{Proposition 7 (Harer-Zagier series formula [8, 9]) The generating function}

$$
E(x, y):=1+2 x y+2 \sum_{g \geq 0, n>0} \frac{\epsilon_{g}(n)}{(2 n-1) ! !} y^{n+1} x^{n+1-2 g} \text { is given by } E(x, y)=\left(\frac{1+y}{1-y}\right)^{x} \text {. }
$$

\subsection{Harer-Zagier recurrence formula}

Elementary algebraic manipulations on the expression of $E(x, y)$ yield a very simple recurrence satisfied by $\epsilon_{g}(n)$, known as the Harer-Zagier recurrence formula (stated in Proposition 10 hereafter). We now show that the model of $\mathrm{C}$-decorated trees makes it possible to derive this recurrence directly from a combinatorial isomorphism, that generalizes Rémy's beautiful bijection for plane trees [13].

It is convenient here to consider C-decorated trees as unlabelled structures: precisely we see a Cdecorated tree as a plane tree where the vertices are partitioned into parts of odd size, where each part carries a sign + or - , and such that the vertices in each part are cyclically ordered (the C-permutation can be recovered by numbering the vertices of the tree according to a left-to-right depth-first traversal), think of Figure 1 (c) where the labels have been taken out. We denote by $\mathcal{P}(n)=\mathcal{E}_{0}(n)$ the set of plane trees with $n$ edges, and by $\mathcal{P}^{\mathrm{v}}(n)\left(\right.$ resp. $\left.\mathcal{P}^{\mathrm{c}}(n)\right)$ the set of plane trees with $n$ edges where a vertex (resp. a corner) is marked. Rémy's procedure, shown in Figure 3 , realizes the isomorphism $\mathcal{P}^{\mathrm{v}}(n) \simeq 2 \mathcal{P}^{\mathrm{c}}(n-1)$, or equivalently

$$
(n+1) \mathcal{P}(n) \simeq 2(2 n-1) \mathcal{P}(n-1) .
$$

Let $\mathcal{T}_{g}^{\mathrm{v}}(n)$ be the set of $\mathrm{C}$-decorated trees from $\mathcal{T}_{g}(n)$ where a vertex is marked. Let $\mathcal{A}$ (resp. $\mathcal{B}$ ) be the subset of objects in $\mathcal{T}_{g}^{\mathrm{v}}(n)$ where the signed cycle containing the marked vertex has length 1 (resp. length greater than 1). Let $\gamma \in \mathcal{T}_{g}^{\mathrm{V}}(n)$, with $n \geq 1$. If $\gamma \in \mathcal{A}$, record the sign of the 1-cycle containing $v$ and then apply the Rémy's procedure to the plane tree with respect to $v$ (so as to delete $v$ ). This reduction, which does not change the genus, yields $\mathcal{A} \simeq 2 \cdot 2(2 n-1) \mathcal{T}_{g}(n-1)$. If $\gamma \in \mathcal{B}$, let $c$ be the cycle containing the marked vertex $v ; c$ is of the form $\left(v, v_{1}, v_{2}, \ldots, v_{2 k}\right)$ for some $k \geq 1$. Move $v_{1}$ and $v_{2}$ out of $c$ (the successor of $v$ becomes the former successor of $v_{2}$ ). Then apply the Rémy's procedure twice, firstly with respect to $v_{1}$ (on a plane tree with $n$ edges), secondly with respect to $v_{2}$ (on a plane tree with $n-1$ edges). This reduction, which decreases the genus by 1 , yields $\mathcal{B} \simeq 2(2 n-1) 2(2 n-3) \mathcal{T}_{g-1}^{\mathrm{v}}(n-2)$, hence $\mathcal{B} \simeq 4(n-1)(2 n-1)(2 n-3) \mathcal{T}_{g-1}(n-2)$. Since $\mathcal{T}_{g}^{\mathrm{v}}(n)=\mathcal{A}+\mathcal{B}$ and $\mathcal{T}_{g}^{\mathrm{v}}(n) \simeq(n+1) \mathcal{T}_{g}(n)$, we finally obtain the isomorphism

$$
(n+1) \mathcal{T}_{g}(n) \simeq 4(2 n-1) \mathcal{T}_{g}(n-1)+4(n-1)(2 n-1)(2 n-3) \mathcal{T}_{g-1}(n-2),
$$

which holds for any $n \geq 1$ and $g \geq 0$ (with the convention $\mathcal{T}_{g}(n)=\emptyset$ if $g$ or $n$ is negative). Since $2^{n+1} \mathcal{E}_{g}(n) \simeq \mathcal{T}_{g}(n)$, we recover: 
Proposition 8 (Harer-Zagier recurrence formula $[\mathbf{8}, 9])$ The coefficients $\epsilon_{g}(n)$ satisfy the following recurrence relation valid for any $g \geq 0$ and $n \geq 1$ (with $\epsilon_{0}(0)=1$ and $\epsilon_{g}(n)=0$ if $g<0$ or $n<0$ ):

$$
(n+1) \epsilon_{g}(n)=2(2 n-1) \epsilon_{g}(n-1)+(n-1)(2 n-1)(2 n-3) \epsilon_{g-1}(n-2) .
$$

To the best of our knowledge this is the first proof of the Harer-Zagier recurrence formula that directly follows from a combinatorial isomorphism. The isomorphism (3) also provides a natural extension to arbitrary genus of Rémy's isomorphism (2).

\subsection{Refined enumeration of bipartite unicellular maps}

In this paragraph, we explain how to recover a formula due to Goupil and Schaeffer [7, Theorem 2.1] from our bijection. Let us first give a few definitions. A graph is bipartite if its vertices can be colored in black and white such that each edge connects a black and a white vertices. If the graph has a root-vertex $v$, then $v$ is required to be black; and if the graph is also connected, then such a bicoloring of the vertices is unique. From now on, a connected bipartite graph with a root-vertex is assumed to be endowed with this canonical bicoloring.

The degree distribution of a map/graph is the sequence of the degrees of its vertices taken in decreasing order (it is a partition of $2 n$, where $n$ is the number of edges). If we consider a bipartite map/graph, we can consider separately the white vertex degree distribution and the black vertex degree distribution, which are two partitions of $n$.

Let $\ell, m, n$ be positive integers such that $n+1-\ell-m$ is even. Fix two partitions $\lambda, \mu$ of $n$ of respective lengths $\ell$ and $m$. We call $\operatorname{Bi}(\lambda, \mu)$ the number of bipartite unicellular maps, with white (resp. black) vertex degree distribution $\lambda$ (resp. $\mu$ ). The corresponding genus is $g=(n+1-\ell-m) / 2$. It will be convenient to change a little bit the formulation of the problem and to consider labelled maps instead of the usual non-labelled maps: we call a labelled map a map whose vertices are labelled with integers $1,2, \cdots$. If the map is bipartite, we require instead that the white and black vertices are labelled separately (with respective labels $w_{1}, w_{2}, \cdots$ and $b_{1}, b_{2}, \cdots$ ). The degree distribution(s) of a (bipartite) labelled map with $n$ edges can be seen as a composition of $2 n$ (resp. two compositions of $n$ ). For $\boldsymbol{I}=\left(i_{1}, \cdots, i_{\ell}\right)$ and $\boldsymbol{J}=\left(j_{1}, \cdots, j_{m}\right)$ two compositions of $n$, we denote by $\operatorname{BiL}(\boldsymbol{I}, \boldsymbol{J})$ the number of labelled bipartite unicellular maps with white (resp. black) vertex degree distribution $\boldsymbol{I}$ (resp. $\boldsymbol{J}$ ). The link between $\operatorname{Bi}(\lambda, \mu)$ and $\operatorname{BiL}(\boldsymbol{I}, \boldsymbol{J})$ is straightforward: $\operatorname{BiL}(\boldsymbol{I}, \boldsymbol{J})=m_{1}(\lambda) ! m_{2}(\lambda) ! \cdots m_{1}(\mu) ! m_{2}(\mu) ! \cdots \operatorname{Bi}(\lambda, \mu)$, where $\lambda$ and $\mu$ are the sorted versions of $\boldsymbol{I}$ and $\boldsymbol{J}$. We now recover the following formula:

\section{Proposition 9 (Goupil and Schaeffer [7, Theorem 2.1]) :}

$$
\begin{aligned}
& \operatorname{BiL}(\boldsymbol{I}, \boldsymbol{J})=2^{-2 g} \cdot n \cdot\left(\ell+2 g_{1}-1\right) !\left(m+2 g_{2}-1\right) ! \\
& \quad \sum_{g_{1}+g_{2}=g} \sum_{\substack{p_{1}+\cdots+p_{\ell}=g_{1} \\
q_{1}+\cdots+q_{m}=g_{2}}} \prod_{r=1}^{\ell} \frac{1}{2 p_{r}+1}\left(\begin{array}{c}
i_{r}-1 \\
2 p_{r}
\end{array}\right) \prod_{r=1}^{m} \frac{1}{2 q_{r}+1}\left(\begin{array}{c}
j_{r}-1 \\
2 q_{r}
\end{array}\right) .
\end{aligned}
$$

Proof: For $g=0$ the formula is simply

$$
\operatorname{BiL}(\boldsymbol{I}, \boldsymbol{J})=n(\ell-1) !(m-1) !
$$


which can easily be established by a bivariate version of the cyclic lemma, see also [5, Theorem 2.2]. (Note, that in that case, the cardinality only depend on the lengths of $\boldsymbol{I}$ and $\boldsymbol{J}$.)

We now prove the formula for arbitrary $g$. Consider some lists $\boldsymbol{p}=\left(p_{1}, \cdots, p_{\ell}\right)$ and $\boldsymbol{q}=\left(q_{1}, \cdots, q_{m}\right)$ of nonnegative integers with total sum $g$ : let $g_{1}=\sum p_{i}$ and $g_{2}=\sum q_{i}$. We say that a composition $\boldsymbol{H}$ refines $\boldsymbol{I}$ along $\boldsymbol{p}$ if $\boldsymbol{H}$ is of the form $\left(h_{1}^{1}, \cdots, h_{1}^{2 p_{1}+1}, \cdots, h_{\ell}^{1}, \cdots, h_{\ell}^{2 p_{\ell}+1}\right)$, with $\sum_{t=1}^{2 p_{r}+1} h_{r}^{t}=i_{r}$ for all $r$ between 1 and $\ell$. Clearly, there are $\prod_{r=1}^{\ell}\left(\begin{array}{c}i_{r}-1 \\ 2 p_{r}\end{array}\right)$ such compositions $\boldsymbol{H}$. One defines similarly a composition $\boldsymbol{K}$ refining $\boldsymbol{J}$ along $\boldsymbol{q}$.

Consider now the set of labelled bipartite plane trees of vertex degree distributions $\boldsymbol{H}$ and $\boldsymbol{K}$, where $\boldsymbol{H}$ (resp. $\boldsymbol{K})$ refines $\boldsymbol{I}$ (resp. $\boldsymbol{J}$ ) along $\boldsymbol{p}$ (resp. $\boldsymbol{q})$. By (5), there are $n \cdot\left(\ell+2 g_{1}-1\right) !\left(m+2 g_{2}-1\right)$ ! trees for each pair $(\boldsymbol{H}, \boldsymbol{K})$, so in total, with $\boldsymbol{I}, \boldsymbol{J}, \boldsymbol{p}$ and $\boldsymbol{q}$ fixed, the number of such trees is:

$$
n \cdot\left(\ell+2 g_{1}-1\right) !\left(m+2 g_{2}-1\right) ! \prod_{r=1}^{\ell}\left(\begin{array}{c}
i_{r}-1 \\
2 p_{r}
\end{array}\right) \prod_{r=1}^{m}\left(\begin{array}{c}
j_{r}-1 \\
2 q_{r}
\end{array}\right) .
$$

As the parts of $\boldsymbol{H}$ (resp. $\boldsymbol{K}$ ) are naturally indexed by pairs of integers, we can see these trees as labelled by the set $\left\{w_{r}^{t} ; 1 \leq r \leq \ell, 1 \leq t \leq 2 p_{r}+1\right\} \sqcup\left\{b_{r}^{t} ; 1 \leq r \leq m, 1 \leq t \leq 2 q_{r}+1\right\}$. There is a canonical permutation of the vertices of the trees with cycles of odd sizes and which preserves the bicoloring: just send $w_{r}^{t}$ to $w_{r}^{t+1}$ (resp. $b_{r}^{t}$ to $b_{r}^{t+1}$ ), where $t+1$ is meant modulo $2 p_{r}+1$ (resp. $2 q_{r}+1$ ). If we additionally put a sign on each cycle, we get a C-decorated tree (with labelled cycles) that corresponds to a labelled bipartite map with white (resp. black) vertex degree distribution $\boldsymbol{I}$ (resp. $\boldsymbol{J}$ ). Conversely, to recover a labelled bipartite plane tree from such a C-decorated tree, one has to choose in each cycle which vertex gets the label $w_{r}^{1}$ or $b_{r}^{1}$, and one has to forget the signs of the $(n+1-g)$ cycles. This represents a factor $2^{n+1-2 g}\left(\prod_{r=1}^{\ell}\left(2 p_{r}+1\right) \prod_{r=1}^{m}\left(2 q_{r}+1\right)\right)^{-1}$.

Multiplying (6) by the above factor, and summing over all possible sequences $\boldsymbol{p}$ and $\boldsymbol{q}$ of total sum $g$, we conclude that the number of $\mathrm{C}$-decorated trees associated with labelled bipartite unicellular maps of white (resp. black) vertex degree distibution $\boldsymbol{I}$ (resp. $\boldsymbol{J}$ ), is equal to $2^{n+1}$ times the right-hand side of (4). By Theorem 5, this number is also equal to $2^{n+1} \operatorname{BiL}(\boldsymbol{I}, \boldsymbol{J})$. This ends the proof of Proposition 9

This is the first combinatorial proof of (4) (the proof by Goupil and Schaeffer involves representation theory of the symmetric group). Moreover, the authors of [7] found surprising that "the two partitions contribute independently to the genus". With our approach, this is very natural, since the cycles are carried independently by white and black vertices.

\subsection{Summation formulas for colored maps}

We now recover Harer-Zagier's summation formula [8, 9] (which can also be very easily derived from the expression of $E(x, y)$ ). In contrast to the formulas presented so far, this one has already been given combinatorial proofs [10, 6, 1] using different bijective constructions, but we want to insist on the fact that our construction gives bijective proofs for all the formulas in a unified way.

Proposition 10 (Harer-Zagier summation formula [8, 9]) Let $A(v ; n)$ be the number of unicellular maps with $n$ edges and $v$ vertices. Then for $n \geq 1$

$$
\sum_{v} A(v ; n) x^{v}=(2 n-1) ! ! \sum_{r \geq 1} 2^{r-1}\left(\begin{array}{c}
n \\
r-1
\end{array}\right)\left(\begin{array}{l}
x \\
r
\end{array}\right) .
$$


Proof: It suffices to prove that the number $A_{r}(n)$ of unicellular maps with $n$ edges, each vertex having a color in [1..r] , and each color in $[1 . . r]$ being used at least once, is given by $A_{r}(n)=(2 n-1) ! ! 2^{r-1}\left(\begin{array}{c}n \\ r-1\end{array}\right)$. Consider a C-decorated tree with $n$ edges, where each (signed) cycle has a color in [1..r], and such that each color in $[1 . . r]$ is used by at least one cycle. Each of the $r$ colors yields a (non-empty) C-permutation, which can be represented as a signed sequence, according to Lemma 3 Then one can concatenate these $r$ signed sequences into a unique sequence $S$ of length $n+1$, together with $r$ signs and a subset of $r-1$ elements among the $n$ elements from position 2 to $n+1$ in $S$ (in order to recover from $S$ the $r$ signed sequences). For instance if $r=3$ and if the signed sequences corresponding respectively to colors $1,2,3$ $\operatorname{are}^{+}(3,9,4),-(5,8,6,2)$, and ${ }^{-}(1,7)$, then the concatenated sequence is $(3,9,4,5,8,6,2,1,7)$, together with the 3 signs $(+,-,-)$ and the two selected elements $\{5,1\}$. Hence the number of such C-decorated trees is $(n+1) ! 2^{r}\left(\begin{array}{c}n \\ r-1\end{array}\right)$, and by Theorem 5 ,

$$
A_{r}(n)=2^{-n-1} \operatorname{Cat}(n)(n+1) ! 2^{r}\left(\begin{array}{c}
n \\
r-1
\end{array}\right)=(2 n-1) ! ! 2^{r-1}\left(\begin{array}{c}
n \\
r-1
\end{array}\right) .
$$

The papers [14, 11] contain other summation formulas, that deal with colored bipartite maps, taking the number of colors or the degree distributions into account. They can all be recovered from our bijection. The proofs follow roughly the same guideline, but are omitted here for brevity.

\section{Computing Stanley character polynomials}

We now consider the following enumerative problem. For $n$ a fixed integer, we would like to compute the generating series $F_{n}\left(p_{1}, p_{2}, \cdots ; q_{1}, q_{2}, \cdots\right)=\sum \mathrm{wt}(M, \varphi)$ of pairs $(M, \varphi)$ where $M$ is a rooted bipartite unicellular map with $n$ edges, and $\varphi$ is a mapping from the vertex set $V_{M}$ of $M$ to positive integers, satisfying the following order condition: for each edge e of $M$, one has $\varphi\left(b_{e}\right) \geq \varphi\left(w_{e}\right)$, where $b_{e}$ and $w_{e}$ are respectively the black and white extremities of $e$. The weight of such a pair is $\operatorname{wt}(M, \varphi):=$ $\prod_{v \in V_{M}^{\circ}} p_{\varphi(i)} \prod_{v \in V_{M}^{\bullet}} q_{\varphi(i)}$, where $V_{M}^{\bullet}$ and $V_{M}^{\circ}$ are respectively the sets of black (resp. white) vertices of $M$.

Our motivation comes from representation theory of the symmetric group. This topic is linked to map enumeration by the following formula [4]. Let $\boldsymbol{p}=p_{1}, \cdots, p_{r}$ and $\boldsymbol{q}=q_{1}, \cdots, q_{r}$ be two finite lists of positive integers of the same length. Then the evaluation $F_{n}\left(p_{1}, \cdots, p_{r}, 0, \cdots ; q_{1}, \cdots, q_{r}, 0, \cdots\right)$ of the generating series considered above is equal to $L(L-1) \cdots(L-n+1) \hat{\chi}^{\lambda}((12 \cdots n))$, where:

- $\lambda$ is the partition with $p_{1}$ parts equal to $q_{1}+\cdots+q_{r}, p_{2}$ parts equal to $q_{2}+\cdots+q_{r}$, and so on...

- $L=\sum_{1 \leq i \leq j \leq r} p_{i} q_{j}$ is its number of boxes ;

- $\hat{\chi}^{\lambda}$ is the normalized character of the irreducible representation of $S_{L}$ associated to $\lambda$;

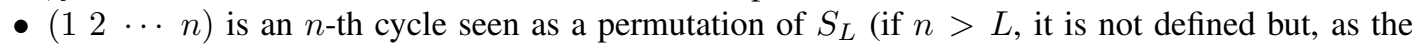
numerical factor is 0 , it is not a problem).

Our main bijection allows us to express the generating series $F_{n}$ in terms of the corresponding generating series for plane trees. A. Rattan has proved [12] that this generating series is the $n+1$-th free cumulant $R_{n+1}$ of the transition measure of $\lambda$ (as $\lambda$ depends on $\boldsymbol{p}$ and $\boldsymbol{q}, R_{n+1}$ can be seen as a series in $\boldsymbol{p}$ and $\boldsymbol{q}$ ). Free cumulants have become in the last few years an important tool in (asymptotic) representation theory of the symmetric groups, see for example the work of P. Biane [2].

Let us define an operator $D$ by $D\left(x^{k}\right):=\sum_{g \geq 0} c_{g}(k) x^{k-2 g}=k ! \sum_{r=1}^{k} 2^{r}\left(\begin{array}{c}k-1 \\ r-1\end{array}\right)\left(\begin{array}{l}x \\ r\end{array}\right), D$ being extended multiplicatively to monomials in distinct variables, and then extended linearly to multivariate polynomials and series (in particular, series in the variables $\boldsymbol{p}$ and $\boldsymbol{q}$ ). 
Theorem 11 For any $n \geq 1$, one has $2^{n+1} F_{n}=D\left(R_{n+1}\right)$.

Proof: A pair $(M, \varphi)$ as above corresponds by the bijection of Section 2 to a bipartite $C$-decorated tree $T$, together with a function $\varphi: V_{T} \rightarrow \mathbb{N}$ which fulfills the order condition and such that all vertices in a given cycle have the same image by $\varphi$. The result follows directly.

The free cumulant $R_{n+1}$ is the compositional inverse of an explicit series [12]. Hence Theorem 11 ] gives an efficient, easily implemented way of computing Stanley character polynomials $F_{n}$.

\section{References}

[1] O. Bernardi. An analogue of the Harer-Zagier formula for unicellular maps on general surfaces. arXiv:1011.2311, 2010.

[2] P. Biane. Representations of symmetric groups and free probability. Adv. Math., 138(1):126-181, 1998.

[3] G. Chapuy. A new combinatorial identity for unicellular maps, via a direct bijective approach. Advances in Applied Mathematics, 47(4):874 - 893, 2011.

[4] V. Féray. Stanley's formula for characters of the symmetric group. Ann. Comb., 13(4):453-461, 2010.

[5] I. P. Goulden and D. M. Jackson. The combinatorial relationship between trees, cacti and certain connection coefficients for the symmetric group. European J. Combin., 13(5):357-365, 1992.

[6] I. P. Goulden and A. Nica. A direct bijection for the Harer-Zagier formula. J. Comb. Theory, Ser. A, 111(2):224238, 2005.

[7] A. Goupil and G. Schaeffer. Factoring $n$-cycles and counting maps of given genus. European J. Combin., 19(7):819-834, 1998.

[8] J. Harer and D. Zagier. The Euler characteristic of the moduli space of curves. Invent. Math., 85:457-486, 1986.

[9] S. K. Lando and A. K. Zvonkin. Graphs on Surfaces and Their Applications. Springer, 2004.

[10] B. Lass. Démonstration combinatoire de la formule de Harer-Zagier. C. R. Acad. Sci. Paris, 333, Série I:155160, 2001.

[11] A. Morales and E. Vassilieva. Bijective enumeration of bicolored maps of given vertex degree distribution. DMTCS Proceedings, AK:661-672, 2009.

[12] A. Rattan. Stanley's character polynomials and coloured factorizations in the symmetric group. J. Combin. Theory Ser. A, 114(4):535-546, 2008.

[13] J.-L. Rémy. Un procédé itératif de dénombrement d'arbres binaires et son application à leur génération aléatoire. RAIRO Inform. Théor., 19(2):179-195, 1985.

[14] G. Schaeffer and E. A. Vassilieva. A bijective proof of Jackson's formula for the number of factorizations of a cycle. J. Comb. Theory, Ser. A, 115(6):903-924, 2008.

[15] T. R. S. Walsh and A. B. Lehman. Counting rooted maps by genus. I. J. Combin. Theory Ser. B, 13:192-218, 1972. 